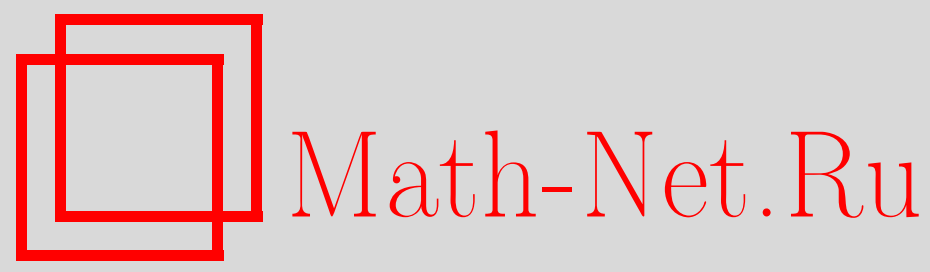

Г. Л. Литвинов, В. П. Маслов, Г. Б. Шпиз, Нецифровая реализация арифметики вещественных чисел средствами квантовых компьютерных сред, Матем. заметки, 2001, том 70, выпуск 1, 59-67

DOI: https://doi.org/10.4213/mzm719

Использование Общероссийского математического портала Math-Net.Ru подразумевает, что вы прочитали и согласны с пользовательским соглашением http://www . mathnet.ru/rus/agreement

Параметры загрузки:

IP : 54.197 .130 .99

26 апреля 2023 г., 16:45:06

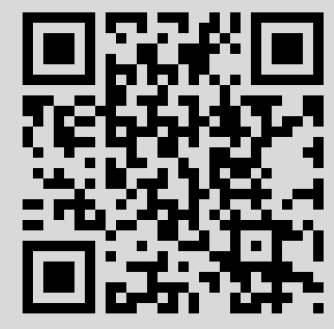




\title{
НЕЦИФРОВАЯ РЕАЛИЗАЦИЯ АРИФМЕТИКИ ВЕЩЕСТВЕННЫХ ЧИСЕЛ СРЕДСТВАМИ КВАНТОВЫХ КОМПЬЮТЕРНЫХ СРЕД
}

\author{
Г. Л. Литвинов, В.П. Маслов, Г. Б. Шпиз
}

\begin{abstract}
В рамках модели квантовых компьютерных сред описана нецифровая реализация арифметики вещественных чисел. Для этой модели элементарной ячейкой памяти является ансамбль $q$-битов (квантовых битов). Оказывается, что для запоминания произвольного вещественного числа достаточно четырех таких ансамблей и арифметические операции могут быть реализованы фиксированными квантовыми схемами.

Библиограбия: 23 названия.
\end{abstract}

Введение. В заметке описана реализация арифметики вещественных чисел в рамках модели квантовой компютерной среды (KKC), которая является расширением стандартной модели квантового компьютера. Элементарной “ячейкой" памяти такой системы является ансамбль квантовых битов (q-битов). Оказывается, что для хранения произвольного вещественного числа достаточно четырех таких ансамблей и арифметические операции реализуются за фиксированное число “тактов”. При этом число представляется в нецифровой форме. Представление числа в цифровой форме (например, в виде двоичной или десятичной дроби) является отдельной задачей статистического оценивания. Другой подход к квантовым вычислениям над непрерывными величинами представлен, например, в [1], [2].

1. Стандартная модель квантовых вычислений. Идея квантовых вычислений первоначально была высказана в работах П. Бениофа [3], [4], Ю.И. Манина [5], Р. Фейнмана [6], [7] и А. Переса [8]. В работе Фейнмана [7] эта идея обсуждалась в деталях. Д. Дейч [9] дал общее формальное определение так назьваемой квантовой машины Тьюринга. В работе [10] он представил другую (эквивалентную, но более удобную) модель, которую принято считать стандартной.

Напомним некоторые основные понятия стандартной модели квантовых вычислений, предложенной в [11]-[13] (подробное изложение см. в этих статьях, а также, например, в [14]), и введем терминологию, используемую в дальнейшем.

Пусть $X$ - конечное множество. Обозначим через $\mathscr{B}(X)$ множество всех булевых функций на $X$, принимающих значения 0 или 1 , а через $\mathscr{H}(X)$ комплексноегильбертово

Работа выполнена при финансовой поддержке Российского фонда фундаментальных исследований, грант № 96-01-01544, и Института математической физики Эрвина Шрёдингера (Вена). 
пространство с ортонормированньм базисом $\mathscr{B}(X)$; таким образом, если $X$ содержит $n$ элементов, то $\mathscr{H}(X)$ - конечномерное гильбертово пространство и его размерность равна $2^{n}$. Пусть $\mathbf{L}(X)$ - алгебра всех линейных ограниченных операторов в $\mathscr{H}(X)$, $\mathbf{U}(X)$ - группа унитарных операторов в $\mathscr{H}(X)$, а $\mathbf{D}(X)$ - множество всех операторов плотности, т.е. положительных самосопряженных операторов в $\mathscr{H}(X)$ со следом, равным 1.

Если $\Delta$ - множество битов, образующих память классического компьютера, то состояния этой памяти описываются элементами из $\mathscr{B}(\Delta)$. Если же $\Delta$ - множество $q$-битов, образуюших квантовую память квантового компююера, то (смешанные) состояния памяти описьваются элементами из $\mathbf{D}(\Delta)$. Разумеется, чистые состояния описываются элементами из $\mathscr{H}(\Delta)$ (с точностью до ненулевого числового коэффициента).

Наделенное линейным порядком подмножество $X$ в $\Delta$ называется регистром. Чистые состояния регистра $X$ соответствуют элементам из $\mathscr{H}(X)$; общие смешанные состояния регистра $X$ соответствуют элементам из $\mathbf{D}(X)$, т.е. операторам плотности в $\mathscr{H}(X)$. В частности, любой $q$-бит является регистром. В этом случае $X$ состоит из единственного элемента и пространство $\mathscr{H}(X)$ двумерно. Если $X$ состоит из $n q$-битов, то $\mathscr{H}(X)$ является тензорньм произведением $n$ двумерных гильбертовых пространств, соответствующих каждому $q$-биту.

Любой $q$-бит имеет два базовых состояния: $|0\rangle$ и $|1\rangle$ (используются хорошо известные обозначения Дирака). Считается, что квантовая память может быть инициализирована произвольным состоянием, которое является тензорным произведением базовых состояний $q$-битов, образующих эту память. Такие состояния называются классическими.

Унитарный оператор $U \in \mathbf{U}(X)$ определяет преобразование $S \mapsto S^{U}$ на множестве $\mathbf{D}(X)$ состояний регистра $X \subset \Delta$ по формуле $S^{U}=U S U^{-1}$. Будем говорить, что унитарньй оператор $U \in \mathbf{U}(\Delta)$ сосредоточен на регистре $X \subset \Delta$, если он имеет вид $U_{X} \otimes \operatorname{id}_{Z}$, где $U_{X} \in \mathbf{U}(X), Z=\Delta \backslash X, \operatorname{a~id}_{Z}$ - тождественный оператор в $\mathscr{H}(Z)$. Соответственно унитарньй оператор $U_{X} \in \mathbf{U}(X)$, где $X \subset \Delta$, будем рассматривать и как оператор вида $U_{X} \otimes \operatorname{id}_{Z}$ из $\mathbf{U}(\Delta)$, где $Z=\Delta \backslash X$. Минимальньй регистр, на котором сосредоточен унитарный оператор $U \in \mathbf{U}(\Delta)$, будем называть носителем $U$ и обозначать $\operatorname{supp}(U)$.

Квантовый компютер вьполняет унитарные преобразования в $\mathscr{H}(\Delta)$. Предполагается, что за один такт вьполняется некоторое элементарное унитарное преобразование, причем имеется конечный набор (базис) таких элементарных операторов, называемых логическими гейтами или просто гейтами. Предполагается, что каждьй гейт имеет короткий носитель (обычно состоящий из одного или двух $q$-битов). Комбинации этих гейтов определяют квантовые схемы (quantum circuits).

Любая биекция $\sigma$ множества классических состояний $\mathscr{B}(X)$ на себя приводит к перестановке элементов ортонормированного базиса в $\mathscr{H}(X)$ и порождает унитарньй оператор $\widehat{\sigma} \in \mathbf{U}(X)$. Операторы (преобразования) этого типа называются классическими.

ПримеР 1. На однобитовом регистре $\{x\}$ перестановка $|0\rangle \mapsto|1\rangle,|1\rangle \mapsto|0\rangle$ задает оператор отричания (или оператор NOT), обозначаемьй через $\neg x$.

ПримеР 2. Другим важным примером является так назьваемьй оператор controlled NOT (или CNOT; см., например, [7]). На двухбитовом регистре $X$ этот оператор определен биекцией $\tau=\tau_{X}:|x, y\rangle \mapsto|x, x \oplus y\rangle$, где $\oplus$ обозначает сложение по модулю 2 . 
Оператор $\tau$ позволяет для классических состояний из $\mathscr{B}(X)$ копировать содержимое первого бита во второй, если второй бит имеет нулевое начальное значение. Разумеется, аналогичный оператор можно определить для пары любых регистров одинаковой длины, применяя $\tau$ к каждой паре битов.

Как указано вьше, операторы, описанные в этих примерах, можно рассматривать как унитарные операторы из $\mathbf{U}(\Delta)$.

Итак, в стандартной модели квантовых вычислений (смешанные) состояния конечной памяти $\Delta$ задаются операторами плотности из $\mathbf{D}(\Delta)$, а элементарные операции (гейты) определяются набором унитарных операторов, сосредоточенных на коротких регистрах. Алгоритм, реализуемый в рамках этой модели, начинается с инициализации памяти классическим состоянием. Затем применяется последовательность унитарных квантовых гейтов. Наконец, выполняется операция измерения (которая является взаимодействием специального типа между квантовым компютером и внешним физическим устройством). Результатом этой операции измерения является классическое состояние регистра. Соответствующие детали см., например, в [11]-[13].

2. Модель квантовой компьютерной среды. Имеется довольно много различных парадигм и моделей для квантовых компьютерных систем (см., например, [2], [11]-[19]). Будем говорить, что компьютерная среда, включающая систему параллельных квантовых компьютеров (процессоров) и классические компоненты, является квантовой компьютерной средой (сокращенно $\mathrm{KKC}$ ). Рассмотрим удобную для наших целей версию этой модели. Эта КKС имеет память $\Delta$, которая образована множеством ансамблей, назьваемых $q$-ансамблями. Грубо говоря, $q$-ансамбль можно рассматривать как поток независимых $q$-битов, а операции над $q$-ансамблями - как независимые воздействия на элементы этих потоков, происходящие в одинаковьх условиях (разумеется, число $q$-битов в $q$-ансамбле конечно, но достаточно велико).

Подобная ситуация возникает, например, для так называемых объемных квантовых вычислений (bulk quantum computation), когда можно манипулировать большим числом неразличимых квантовых компютеров параллельными унитарными операциями (по поводу деталей и реализаций, использующих ядерный магнитный резонанс, см., например, [5]).

Обозначим через $\Delta$ множество всех $q$-ансамблей, образующих память нашей ККС, и обозначим через $\widetilde{\Delta}$ множество всех $q$-битов, принадлежаших этой памяти. Каждый $q$-ансамбль $x \in \Delta$ образует подмножество $\widetilde{\Delta}_{x}$ в $\widetilde{\Delta}$. Будем говорить, что его состояние $\widetilde{S}_{x} \in \mathbf{D}\left(\widetilde{\Delta}_{x}\right)$ допустимо, если $\widetilde{S}_{x}$ является тензорной степенью состояния $S \in \mathbf{D}(\{a\})$, где $a \in \widetilde{\Delta}_{x}$, так что имеется взаимно однозначное соответствие между множеством всех допустимых состояний $q$-ансамбля и множеством всех состояний любого $q$-бита, принадлежащего этому $q$-ансамблю. Каждое смешанное состояние $\widetilde{S} \in \mathbf{D}(\widetilde{\Delta})$ может быть ограничено на любой $q$-ансамбль (посредством формулы частичного следа, см., например, [11], [12] и ниже). Если все такие ограничения допустимы, то будем говорить, что состояние $\widetilde{S}$ допустимо. Обозначим через $\mathrm{D}^{*}(\Delta)$ множество всех допустимых состояний нашей КKС. Будем говорить, что унитарный оператор $U \in \mathbf{U}(\widetilde{\Delta})$ допустим, если $\mathbf{D}^{*}(\Delta)$ эквивалентно относительно действия этого оператора.

Ясно, что соответствующая стандартная модель квантового компьютера может быть вложена в модель $\mathrm{KKC}$ так, что $\mathbf{D}(\Delta)$ и $\mathbf{U}(\Delta)$ соответствуют множествам допустимых 
состояний и допустимых унитарных операторов. Предполагается, что множество всех классических состояний в стандартной модели можно идентифицировать с соответствующим множеством состояний модели ККС. Поэтому каждый алгоритм, реализованньй в рамках стандартной модели, может быть перенесен в модель ККС.

Однако в случае КKС можно построить операцию клонирования (копирования), которая переводит любой $q$-ансамбль в допустимом состоянии в пару $q$-ансамблей так, что каждьй их $q$-бит имеет то же самое состояние. Операцию копирования можно трактовать как разделение исходного q-ансамбля на большие части. Отметим, что в рамках стандартной модели совершенное клонирование невозможно: неизвестное квантовое состояние не может быть клонировано (исключая тот случай, когда состояние уже известно, т.е. существует классическая информация, которая описьвает его). Однако возможно делать приближенные копии. По поводу деталей см., например, [13], [20]-[23].

3. Моделирование арифметики вещественных чисел. Итак, в рамках модели ККС можно реализовать стандартньй квантовый компютер со смешанньми состояниями и операцией клонирования. Ниже нам понадобится получить набор q-битов, приготовленных в идентичных классических состояниях, и манипулировать этими копиями посредством параллельных унитарных операций. Поэтому для простоты будем рассматривать стандартную модель, расширенную операцией клонирования. Эта операция не является квантовой унитарной операцией. Однако мы будем включать ее в квантовые схемы (аналогичный прием был использован в [13] по отношению к операциям измерения). Для такого квантового компьютера ниже представлена реализация арифметики вешественных чисел. При этом скорость выполнения арифметических операций не зависит от сложности операндов (в обычном смысле). В частности, для функции $n \mapsto a^{n}$, где $n \in \mathbb{N}$ и $a$ является произвольным вещественным числом, можно получить полиномиальный алгоритм ее вычисления (по отношению к размеру числа $n$, т.е. $\log n)$, используя хорошо известный стандартньй прием: $a \mapsto a^{2} \mapsto a^{4}=\left(a^{2}\right) \cdot\left(a^{2}\right)$ и т.д.

Обозначим через $\mathscr{B}(n)$ множество $\mathscr{B}(\{1, \ldots, n\})$, а через $\mathscr{H}(n)$ гильбертово пространство $\mathscr{H}(\{1, \ldots, n\})$ (см. обозначения, введенные вьше, в п. 1$)$. Элементы из $\mathscr{B}(1)$ обозначим через $|0\rangle$ и $|1\rangle$, а элементы из $\mathscr{B}(n)$ - через $\left|\alpha_{1}, \ldots, \alpha_{n}\right\rangle$, где $\left|\alpha_{i}\right\rangle \in \mathscr{B}(1)$. Используя обозначения Дирака, элементы из $\mathscr{H}(X)$ будем обозначать через $|x\rangle$ (кет-векторы), а скалярное произведение векторов $|x\rangle$ и $|y\rangle$ - через $\langle x \mid y\rangle$. Выражение $\langle x|$ (бра-вектор) определяет линейный функционал $|y\rangle \mapsto\langle x \mid y\rangle$ на $\mathscr{H}(X)$, а выражение $|a\rangle\langle b|$ - линейньй оператор $|x\rangle \mapsto\langle b \mid x\rangle|a\rangle$. Мы предполагаем, что каждый регистр линейно упорядочен; поэтому $\mathscr{B}(X)$ и $\mathscr{H}(X)$ естественно отождествляются с $\mathscr{B}(n)$ и $\mathscr{H}(n)$, где $n$ - длина регистра $X$ (т.е. число элементов в $X)$.

Пусть $S$ - (смешанное) состояние памяти $\Delta$ и $X \subset \Delta$ является произвольным регистром. Ограничение состояния $S$ на регистр $X$ определяется формулой частичного следа $S \mapsto S(X)=\operatorname{Tr}_{Z}(S) \in \mathbf{D}(X)$, где $Z=\Delta \backslash X$ (см., например, [11], [12]), и мы будем говорить, что $S(X)$ является состоянием регистра $S$. Любое состояние одноточечного регистра $\{x\}$, т.е. $q$-бита, определяется соответствующей матрицей плотности $S=\left(S_{i j}\right)$ по отношению к базису $\mathscr{B}(\{x\})=\{|0\rangle,|1\rangle\}$. При этом компонента $S_{00}$ этой матрицы равна вероятности при измерении найти $q$-бит в состоянии $|0\rangle$; аналогично $S_{11}$ совпадает с вероятностью найти $q$-бит в состоянии $|1\rangle$. Классическим состояниям $X$, т.е. элементам $f \in \mathscr{B}(X)$, соответствуют операторы $\bigotimes_{x \in X}|f(x)\rangle\langle f(x)|$. В частности, 
положим

$$
\mathbf{0}_{X}=\bigotimes_{x \in X}|0\rangle\left\langle 0\left|, \quad \mathbf{1}_{X}=\bigotimes_{x \in X}\right| 1\right\rangle\langle 1| .
$$

Эти операторы соответствуют булевым функциям (классическим состояниям) на $X$, тождественно равным нулю и единице.

Рассмотрим разбиение регистра $X=\bigcup_{i=1}^{n} X_{i}$ на непересекающиеся регистры $X_{i}$. Ясно, что в этом случае пространство $\mathscr{H}(X)$ может быть представлено в виде тензорного произведения $\mathscr{H}(X)=\bigotimes_{i=1}^{n} \mathscr{H}\left(X_{i}\right)$ пространств $\mathscr{H}\left(X_{i}\right)$. Поэтому для любого набора операторов $A_{i} \in \mathbf{L}\left(X_{i}\right)$ существует их тензорное произведение $A=\bigotimes_{i=1}^{n} A_{i} \in$ $\mathbf{L}(X)$. Будем говорить, что этот оператор $A$ разложим относительно разбиения $X=\bigcup_{i=1}^{n} X_{i}$. Заметим, что тензорное произведение операторов плотности является оператором плотности, равно как тензорное произведение унитарньх операторов является унитарным оператором. Будем говорить, что состояние (т.е. элемент из $\mathbf{D}(X) \subset$ $\mathbf{L}(X))$ является (просто) разложимым, если оно разложимо относительно разбиения регистра $X$ на точки, т.е. регистры, состоящие из одного $q$-бита. Отметим, что любое классическое состояние разложимо.

Пусть $A=A_{1} \cup A_{2}$ - разбиение регистра $A$ на непересекающиеся регистры $A_{1}$ и $A_{2}$, $X_{1} \subset A_{1}, X_{2} \subset A_{2}$. Нетрудно проверить, что если состояние $S \in \mathbf{D}(A)$ разложимо относительно разбиения $A=A_{1} \cup A_{2}$, то ограничение этого состояния на регистр $X=X_{1} \cup X_{2}$ разложимо относительно разбиения $X=X_{1} \cup X_{2}$. Будем говорить, что состояние $S \in \mathbf{D}(A)$ разложимо относительно регистра $X \subset \Delta$, если его ограничение $S(X)$ на $X$ разложимо.

Назовем регистр $F$ свободным относительно состояния $S$, а состояние $S$ свободным относительно регистра $F$, если $S=S^{\prime} \otimes \mathbf{0}_{F}$, где $S^{\prime} \in \mathbf{D}(\Delta \backslash F)$. Если $U \in \mathbf{U}(\Delta)$ и регистр $F$ свободен относительно $S$, то регистр $F \backslash \operatorname{supp}(U)$ свободен относительно состояния $S^{U}=U S U^{-1}$.

Теперь мы можем обсудить нашу реализацию арифметики вещественных чисел. Пусть $S$ - состояние регистра $X$; обозначим через $S(x)$ ограничение состояния $S$ на $q$-бит $x \in X$. Предположим, что $X=\left\{x_{1}, x_{2}, x_{3}, x_{4}\right\}$ является регистром, состоящим из четырех $q$-битов, а $S$ - разложимое состояние регистра $X$, причем $S(x)_{i j}$ - соответствующая матрица плотности.

Будем считать, что любое разложимое состояние $S$ четырехбитового регистра $X=$ $\left\{x_{1}, x_{2}, x_{3}, x_{4}\right\}$ моделирует число

$$
r(S)=\frac{\left(S\left(x_{1}\right)\right)_{11}-\left(S\left(x_{2}\right)\right)_{11}}{\left(S\left(x_{3}\right)\right)_{11}-\left(S\left(x_{4}\right)\right)_{11}} .
$$

Разумеется, разные разложимые состояния регистра $X$ могут представлять одно и то же число. Число, представленное состоянием $S$ в виде (1), будем назьвать числом типа real4.

Арифметическая операция $\circledast$ (например, умножение чисел) реализуется некоторой схемой $\mathcal{U}$. Предположим, что $F$ - свободная память и числа записаны в непересекающихся регистрах $A$ и $B$. Допустим, что $S$ - начальное состояние, которое свободно относительно $F$ и разложимо относительно $A$ и $B$. Соответствуюшая схема $\mathcal{U}$ переводит $S$ в такое состояние $\widetilde{S}$, что ограничение $\widetilde{S}(A)$ состояния $S$ на регистр $A$ разложимо 
и $r(\widetilde{S}(A))=r(S(A)) \circledast r(S(B))$. Схема $\mathcal{U}$ является фиксированной конечной комбинацией унитарных операторов из фиксированного набора гейтов. Естественно говорить, что этот набор является системой команд соответствующего арифметического процессора.

Наряду с числами типа real 4 мы будем рассматривать числа типа real1 и real2. Coстояние $S(x)$ однобитового регистра $\{x\}$ представляет число типа real1

$$
r(S(x))=(S(x))_{11},
$$

так что $0 \leqslant r(S(x)) \leqslant 1$.

Пусть $B=\left\{b^{+}, b^{-}\right\}$является двухбитовьг регистром, а $S=S(B)$ - его разложимое состояние; тогда будем говорить, что $S(B)$ представляет следующее вещественное число типа real2:

$$
r(S(B))=\left(S\left(b^{+}\right)\right)_{11}-\left(S\left(b^{-}\right)\right)_{11}=r\left(S\left(b^{+}\right)\right)-r\left(S\left(b^{-}\right)\right) .
$$

Ясно, что $-1 \leqslant r(S(B)) \leqslant 1$, а число $r$ типа real4 можно рассматривать как пару чисел $\left(r^{\prime}, r^{\prime \prime}\right)$ типа real 2 , причем $r=r^{\prime} / r^{\prime \prime}$.

Пусть $S$ - состояние однобитового квантового регистра; тогда будем говорить, что $S$ диагонально, если соответствующая матрица $S_{i j}(i, j=1,2)$ диагональна. Предположим, что $S$ является состоянием произвольного регистра; тогда будем говорить, что состояние $S$ диагонально, если оно разложимо и его ограничение на каждьй $q$-бит из этого регистра является диагональньм. Состояния двух q-битов будем называть әквивалентными, если их матрицы плотности имеют одинаковые диагональные элементы, т.е. состояния эквивалентны, когда они представляют одно и то же число типа real1. Состояния регистров одинаковой длины будем называть әквивалентнымu, если эти регистры и ограничения этих состояний на соответствующие компоненты (q-биты) эквивалентны.

Опишем операцию, преобразующую состояния $q$-битов к эквивалентньм диагональным состояниям за счет использования свободной памяти. Если $\{b\}$ лежит в свободной памяти, то применение этой операции к регистру $X=\{a, b\}$, находящемуся в состоянии $S$, переводит его в такое диагональное состояние $S^{\prime}$, что $S^{\prime}(a)$ и $S^{\prime}(b)$ эквивалентны $S(a)$. Для этого можно использовать оператор CNOT (описанньй в примере 2 , см. выше).

Элементарная проверка показьвает, что справедливо следующее утверждение.

ПРЕДЛОЖЕНИЕ 1. Пусть регистр $X=\{a, b\}$ находится в состоянии $S=S(a) \otimes$ $\mathbf{0}_{\{b\}}, a S^{\tau}=U S U^{-1}$, әде $U=\hat{\tau}$ является классическим оператором СNOT, описанным в примере 2. Тогда $S^{\tau}(a)=S^{\tau}(b)=\left(\delta_{i j}(S(a))_{i j}\right)$, где $\delta_{i j}-$ символ Кронекера, $i, j=1,2$.

\section{4. Реализация арифметических операций.}

4.1. Опишем набор команд (т.е. унитарных операторов), обеспечивающих вьполнение арифметических операций. Сначала опишем две элементарные операции на числах $x$ и $y$ типа real1:

$$
\begin{aligned}
\sigma_{1}: x, y & \mapsto \frac{x+y}{2}, \\
\sigma_{2}: x, y & \mapsto 1-(x+y)+2 x y .
\end{aligned}
$$


Соответствующие унитарные операторы действуют на двухбитовых регистрах $X=$ $\{a, b\}$, т.е. на пространстве $\mathscr{H}(X)=\mathscr{H}(2)$.

Непосредственная проверка показьвает, что если входное состояние $S$ на регистре $X=\{a, b\}$ разложимо, т.е. $S(X)=S(a) \otimes S(b)$, то операция $\sigma_{2}$ реализуется классическим оператором, порожденньм перестановкой элементов ортонормированного базиса в $\mathscr{H}(2)$ :

$$
|1,1\rangle \mapsto|1,1\rangle, \quad|1,0\rangle \mapsto|0,0\rangle, \quad|0,0\rangle \mapsto|0,1\rangle, \quad|0,1\rangle \mapsto|1,0\rangle .
$$

Аналогично, пусть входное состояние $S$ на регистре $X=\{a, b\}$ разложимо и диагонально на каждом из $q$-битов. В этом случае операция $\sigma_{1}$ реализуется унитарным оператором $U$, которьй на базис в $\mathscr{H}(2)$ действует следуюшим образом:

$$
|1,1\rangle \mapsto|1,1\rangle, \quad|0,0\rangle \mapsto|0,0\rangle, \quad|1,0\rangle \mapsto \lambda(|1,0\rangle+|0,1\rangle), \quad|0,1\rangle=\lambda(-|1,0\rangle+|0,1\rangle),
$$

где $\lambda=1 / \sqrt{2}$. Этот оператор $U$ реализует операцию $\sigma_{1}$ лишь в том случае, когда входное состояние $S$ диагонально. Однако оператор $\widehat{\tau}$ (см. вьше пример 2 и предложение 1 ) с $q$-битом из свободной памяти переводит $q$-бит, находящийся в состоянии $S$, в такое диагональное состояние $S^{\prime}$, что $r\left(S^{\prime}\right)=r(S)$. Поэтому, комбинируя операторы $U$ и $\widehat{\tau}$, мы получим реализацию операции $\sigma_{1}$.

Комбинируя операции $\sigma_{1}, \sigma_{2}$ и оператор клонирования, можно вычислить функцию $\mu_{1}(x, y)=\sigma_{1}\left(\sigma_{1}\left(\sigma_{2}(x, y), 0\right), \sigma(x, y)\right)=\frac{(1-(x+y)+2 x y+0) / 2+(x+y) / 2}{2}=\frac{x y}{2}+\frac{1}{4}$.

Таким образом, справедливо

ПРЕДЛОЖЕНИЕ 2. Для всех чисел типа real1 операции среднего арифметического $x, y \mapsto(x+y) / 2$ и смещенного умножения $x, y \mapsto x y / 2+1 / 4$ могут быть реализованы фиксированными квантовыми схемами ${ }^{1}$.

4.2. Покажем теперь, что для чисел типа real 2 справедливо аналогичное

ПРЕДЛоЖЕНИЕ 3. Для всех чисел типа real2 операчии среднего арифметического $x, y \mapsto(x+y) / 2$ и квазиумножения $x, y \mapsto x y / 4$ могут быть реализованы фиксированными квантовыми схемами.

Напомним, что число $z$ типа real2 можно представить как разность $z^{+}-z^{-}$чисел типа real1. Допуская вольность в обозначениях, обозначим операцию среднего арифметического через $\sigma$, а операцию квазиумножения из предложения 3 через $\mu_{2}$.

Реализация указанных в предложении 3 операций задается следуюшими формулами:

$$
\begin{aligned}
& \sigma^{+}(x, y)=\sigma\left(x^{+}, y^{+}\right)=\frac{x^{+}+y^{+}}{2}, \\
& \sigma^{-}(x, y)=\sigma\left(x^{-}, y^{-}\right)=\frac{x^{-}+y^{-}}{2},
\end{aligned}
$$

\footnotetext{
${ }^{1}$ Это означает, что каждая схема является фиксированной комбинацией гейтов (команд).
} 


$$
\begin{aligned}
\mu^{+}(x, y) & =\sigma\left(\mu_{1}\left(x^{+}, y^{+}\right), \mu_{1}\left(x^{-}, y^{-}\right)\right) \\
& =\sigma\left(\frac{x^{+} y^{+}}{2}+\frac{1}{4}, \frac{x^{-} y^{-}}{2}+\frac{1}{4}\right)=\frac{x^{+} y^{+}+x^{-} y^{-}}{4}+\frac{1}{4}, \\
\mu^{-}(x, y) & =\sigma\left(\mu_{1}\left(x^{+}, y^{-}\right), \mu_{1}\left(x^{-}, y^{+}\right)\right) \\
& =\sigma\left(\frac{x^{+} y^{-}}{2}+\frac{1}{4}, \frac{x^{-} y^{+}}{2}+\frac{1}{4}\right)=\frac{x^{+} y^{-}+x^{-} y^{+}}{4}+\frac{1}{4} .
\end{aligned}
$$

В самом деле, $\sigma(x, y)=\sigma^{+}(x, y)-\sigma^{-}(x, y)=(x+y) / 2$ и $\mu_{2}(x, y)=\mu^{+}(x, y)-\mu^{-}(x, y)=$ $\left(x^{+} y^{+}+x^{-} y^{-}-x^{+} y^{-}-x^{-} y^{+}\right) / 4=x y / 4$, что и требовалось. Разумеется, при вычислении приходится пользоваться операциями клонирования.

4.3. Из предложений 2 и 3 легко вьводится следующая

ТЕОремА. Для всех чисел типа real4 операции сложсения, умножсения, вычитания и деления могут быть реализованы фиксированными квантовыми схемами.

Напомним, что число $z$ типа real 4 представляется в виде $z^{\prime} / z^{\prime \prime}$, где $z^{\prime}$ и $z^{\prime \prime}$ являются числами типа real2. Операция умножения выражается следующими формулами:

$$
(x y)^{\prime}=\mu_{2}\left(x^{\prime}, y^{\prime}\right), \quad(x y)^{\prime \prime}=\mu_{2}\left(x^{\prime \prime}, y^{\prime \prime}\right) .
$$

Полусумма $(x+y) / 2$ выражается формулами

$$
\left(\frac{x+y}{2}\right)^{\prime}=\sigma\left(\mu_{2}\left(x^{\prime}, y^{\prime \prime}\right), \mu_{2}\left(x^{\prime \prime}, y^{\prime}\right)\right), \quad\left(\frac{x+y}{2}\right)^{\prime \prime}=\mu_{2}\left(x^{\prime \prime}, y^{\prime \prime}\right)
$$

Наконец, сумма реализуется как умножение полусуммы на число 2, которое легко реализовать как число типа real4.

Из основной формулы (1) ясно, что для любого вещественного числа $r$ и его представления в виде (1) легко построить соответствующие представления для чисел $-r$ и $r^{-1}$; поэтому операции вычитания и деления также могут быть реализованы фиксированными квантовьми схемами.

ЗАмЕчАНИЕ. Отметим, что наши точные определения и конструкции для операций неустойчивы относительно малых возмущений. Однако все элементарные операции непрерьвны, и на практике мы будем иметь дело с приближенными значениями и операциями, ошибками и т.п. (как обычно для операций с вещественными числами). Поэтому необходимо исследовать соответствующие методы для устойчивых по отношению к погрешностям (fault-tolerant) вычислений. Это предмет последующих публикаций.

\section{СПИСОК ЦИТИРОВАННОЙ ЛИТЕРАТУРЫ}

[1] Braunstein S. L. Error correction for continuous quantum variables // Phys. Rev. Lett. 1998. V. 80. № 18. P. 4084-4087.

[2] Lloyd S., Braunstein S. L. Quantum computation over continuous variables // Phys. Rev. Lett. 1999. V. 82. № 8. P. 1784-1787.

[3] Benioff P. The computer as a physical system: a microscopic quantum mechanical Hamiltonian model of computers as represented by Turing Machines // J. Statist. Phys. 1980. V. 22. № 5 . P. 563-591. 
[4] Benioff P. Quantum mechanical Hamiltonian models of Turing machines // J. Statist. Phys. 1982. V. 29. P. 515-546.

[5] Манин Ю. И. Вычислимое и невычислимое. М.: Советское радио, 1980.

[6] Feynman R.P. Simulating physics with computers // Int. J. Theor. Phys. 1982. V. 21. №6/7. P. 467-488.

[7] Feynman R. P. Quantum Mechanical Computers // Optic News. 1985. V. 11. P. 11-20; // Foundations of Physics. 1986. V. 16. №6. P. 507-531.

[8] Peres A. Reversible logic and quantum computers // Phys. Rev. A. 1985. V. 32. P. 3266-3276.

[9] Deutsch D. Quantum theory, the Church-Turing principle and the universal quantum computer // Proc. Roy. Soc. London. Ser. A. 1985. V. 400. P. 97-117.

[10] Deutsch D. Quantum computational networks // Proc. Roy. Soc. London. Ser. A. 1989. V. 425. P. 73-90.

[11] Kitaev A. Yu. Quantum measurements and the abelian stabilizer problem // E-print quant-ph/9511026, 1995.

[12] Китаев А. Ю. Квантовые вычисления: алгоритмы и исправление ошибок // УМН. 1997. T. 52. №6. C. 53-112.

[13] Aharonov D., Kitaev A., Nisan N. Quantum circuits with mixed states // E-print quant-ph/9806029, 1998.

[14] Steane A. Quantum computing // Rep. Prog. Phys. 1998. V. 61. P. 117-173.

[15] Knill E., Chuang I., Laflame R. Effective pure states for bulk quantum computation // E-print quant-ph/9706053, 1997.

[16] Benjamin S. C., Johnson N. F. Structures for data processing in the quantum regime // E-print quant-ph/9802127, 1998.

[17] Ekert A. K. Distributed quantum computations over noisy channels // E-print quant-ph/ 9803017, 1998.

[18] Ozhigov Yu. Quantum computers speed up classical with probability zero // E-print quant-ph/9803064, 1998.

[19] Moore C., Nilsson M. Parallel quantum computation and quantum codes // E-print quant-ph/9808027, 1998.

[20] Barnum H. Noncommuting mixed states cannot be broadcast // Phys. Rev. Lett. 1996. V. 76. P. 2818-2821.

[21] Buzek V., Hillary M. Quantum copying beyond the no-cloning theorem // Phys. Rev. Lett. A. 1996. V. 54. P. 1844-1862.

[22] Gisin N., Massar S. Optimal quantum cloning machines // Phys. Rev. Lett. 1997. V. 79. P. 2153-2156.

[23] Bruss D., Ekert A., Macchiavello C. Optimal universal quantum cloning and state estimation // Phys. Rev. Lett. 1998. V. 81. P. 2598-2601.

(Г. Л. Литвинов, Г. Б. Шпиз) Международный центр "Софус Ли"

Поступило

(В.П. Маслов) Московский государственный университет им. М. В. Ломоносова

03.11 .1998

E-mail: litvinov@islc.msk.su 